Archive for

Organic Chemistry

Arkivoc 2020, part vi, 220-227

\title{
A novel approach for the synthesis of $\beta$-keto esters: one-pot reaction of carboxylic acids with chlorosulfonyl isocyanate
}

\author{
Ufuk Atmaca ${ }^{\mathrm{a}, \mathrm{b}}$ \\ ${ }^{a}$ Oltu Vocational School, Ataturk University, 25400, Oltu-Erzurum, Turkey \\ ${ }^{b}$ Department of Chemistry, Faculty of Sciences, Atatürk University, 25240, Erzurum, Turkey \\ Email: uatmaca@atauni.edu.tr
}

Received 04-01-2020

Accepted 06-12-2020

Published on line $07-06-2020$

\section{Abstract}

$\beta$-Keto esters were synthesized by direct carboxylation of various 4- and 5-oxo-carboxylic acid derivatives in the presence of chlorosulfonyl isocyanate in excellent yield under mild conditions. Additionally, optimization conditions were examined for synthesis $\beta$-keto esters. Finally, it has been found that trifluoroacetic acid is efficient in DCM under optimized conditions. This efficient one-pot novel method is very usefull, fair price and easy to handle of $\beta$-keto esters.

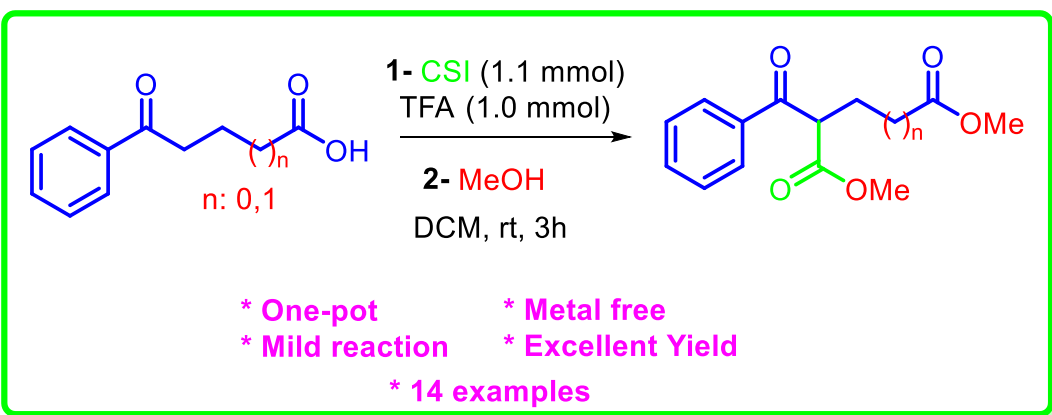

Keywords: $\beta$-Keto ester, chlorosulfonyl isocyanate, carboxylic acid, trifluoroacetic acid, one-pot 


\section{Introduction}

$\beta$-Keto esters are multi-coupling reagents that have both a nucleophilic carbon and an electrophilic carbonyl group. These molecules are very significant intermediates for the synthesis of a wide range of bioactive and natural products such as 1,4-dihydropyridines, arylcoumarins, and 3,4-dihydropyrimidinones, ${ }^{1}$ chokol, ${ }^{2}$ syncarpic acid, ${ }^{3}$ and polyoxamic acid. ${ }^{4}$ Furthermore, they are also used preparation of catalysis, ${ }^{5}$ polymer stabilization, ${ }^{6}$ synthesis of metal microfilms impregnation and liquid crystal preparation technology. ${ }^{7}$ Therefore, various methods have been investigated for the synthesis of their derivatives. ${ }^{1}$ Common methods for the synthesis of $\beta$-keto esters mostly rely on Claisen reaction ${ }^{8}$ and related condensations of enolates with alkyl carbonates and oxalates followed by decarbonylation. ${ }^{9,10}$

a: Synthesis of beta-keto esters by halomethylketones ${ }^{11}$

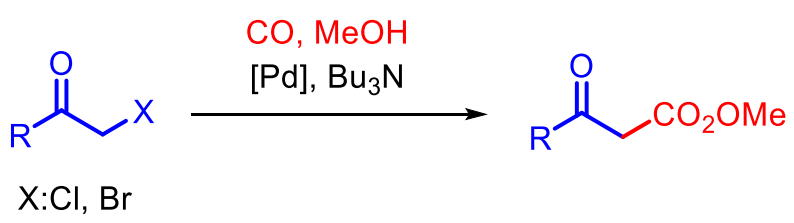

b: Synthesis of beta-keto esters by diazoester and Niobium $(\mathrm{V})$ chloride $^{12}$

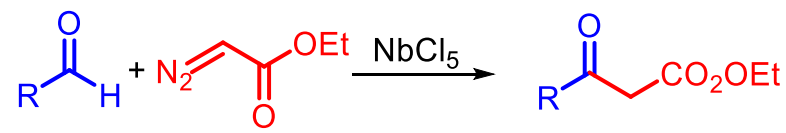

C: Synthesis of beta-keto esters by tertiary amides ${ }^{13}$

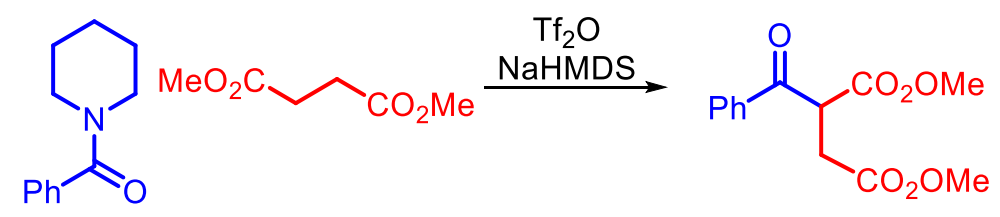

This work:

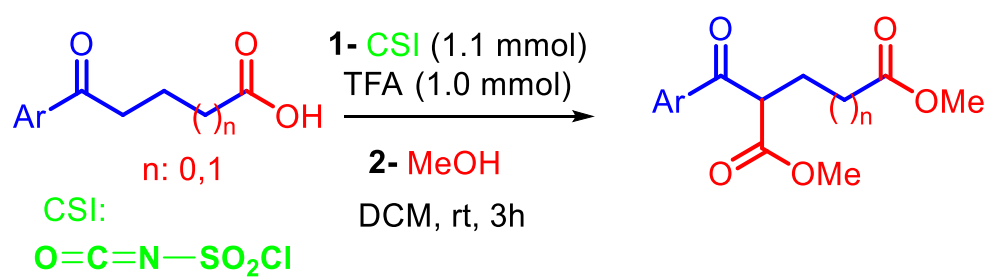

Scheme 1. Methods for the synthesis of $\beta$-keto ester derivatives.

In recent years, with the increasing demands to $\beta$-keto esters, synthesis of these molecules has been reported alkoxycarboxylation of halomethylketones with Pd-catalyzed (Scheme 1a). ${ }^{11}$ Meanwhile, synthesis of $\beta$-keto esters by $\mathrm{C}-\mathrm{H}$ insertion reactions of diazoesters with Niobium(V) chloride catalyzed were reported by the Yadav group (Scheme 1b). ${ }^{12}$ Synthesis of $\beta$-keto esters by aza-Knoevenagel reactions of tertiary amides have been also developed (Scheme 1c). ${ }^{13}$ Recent developments in the synthetic use of CSI are known in the literature. ${ }^{14}$ Previously, we completed reaction of a range of benzoic acid derivatives with CSI that were yielded sulfamate derivatives. ${ }^{15}$ 
In this work, we reported novel and efficient one-pot synthetic method for the synthesis of $\beta$-keto esters (2an) from carboxylic acid derivatives (1a-n) under mild conditions in good yields (Scheme 1).

\section{Results and Discussion}

Initially, the reaction of 5-oxo-5-phenylpentanoic acid with CSI (1a) was chosen that as the model reaction which was carried out in various solvents, acids, bases. The solvent effect was investigated with dichloromethane (DCM) and acetonitrile in the absence of acid and base at room temperature. In these reactions, 2-benzoylpentanedioate (2a) was observed in $54 \%$ and $42 \%$ isolated yield, respectively. The structure of dimethyl 2-benzoylpentanedioate (2a) was verified with ${ }^{1} \mathrm{H}$ - and ${ }^{13} \mathrm{C}-\mathrm{NMR}, \mathrm{IR}$, and HRMS. Then, acid or base effect was examined with the triflic acid (TfOH), trifluoroacetic acid (TFA), triethylamine ( $\mathrm{NEt}_{3}$ ), and pyridine. The use of TfOH gave the dimethyl 2-benzoylpentanedioate (2a) in good yield (entry 2 and 7). The desired product $\mathbf{2 a}$ yielded the use of $\mathrm{NEt}_{3}$, or pyridine in poor yield (entry $4,5,9$, and 10). The desired product dimethyl 2-benzoylpentanedioate (2a) was obtained in $92 \%$ yield, when the reaction was performed in DCM with TFA (entry 8).

Table 1. Model reaction for novel synthesis of dimethyl 2-benzoylpentanedioate (2a)

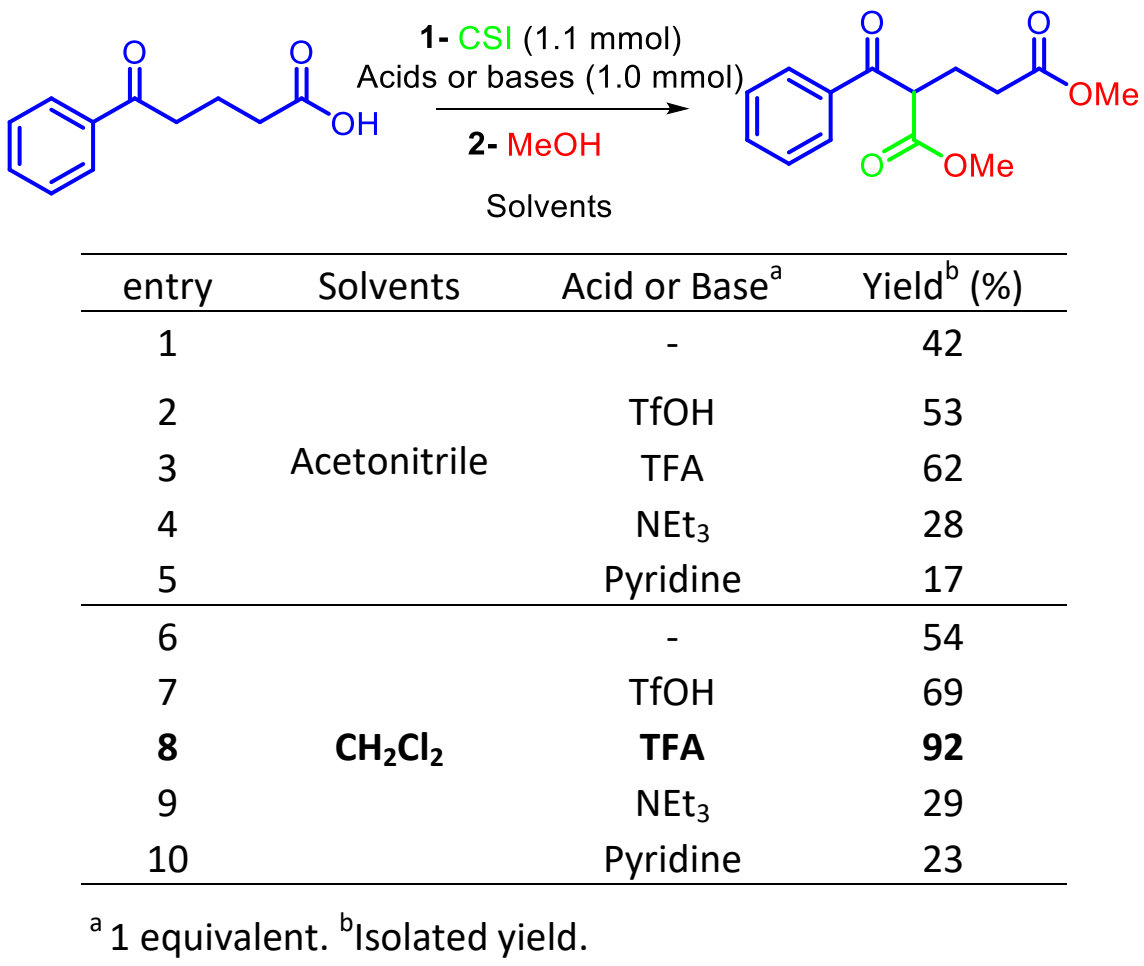

Thus, the optimized reaction condition for alternative and novel intramolecular synthesis of $\beta$-keto esters from 4- and 5-oxocarboxylic acid derivatives was concluded finally to be the use DCM as solvent with TFA as additive under mild conditions at room temperature for $3 \mathrm{~h}$. 
Table 2. Novel synthesis of $\beta$-keto esters from carboxylic acids using TFA ${ }^{a, b, c}$
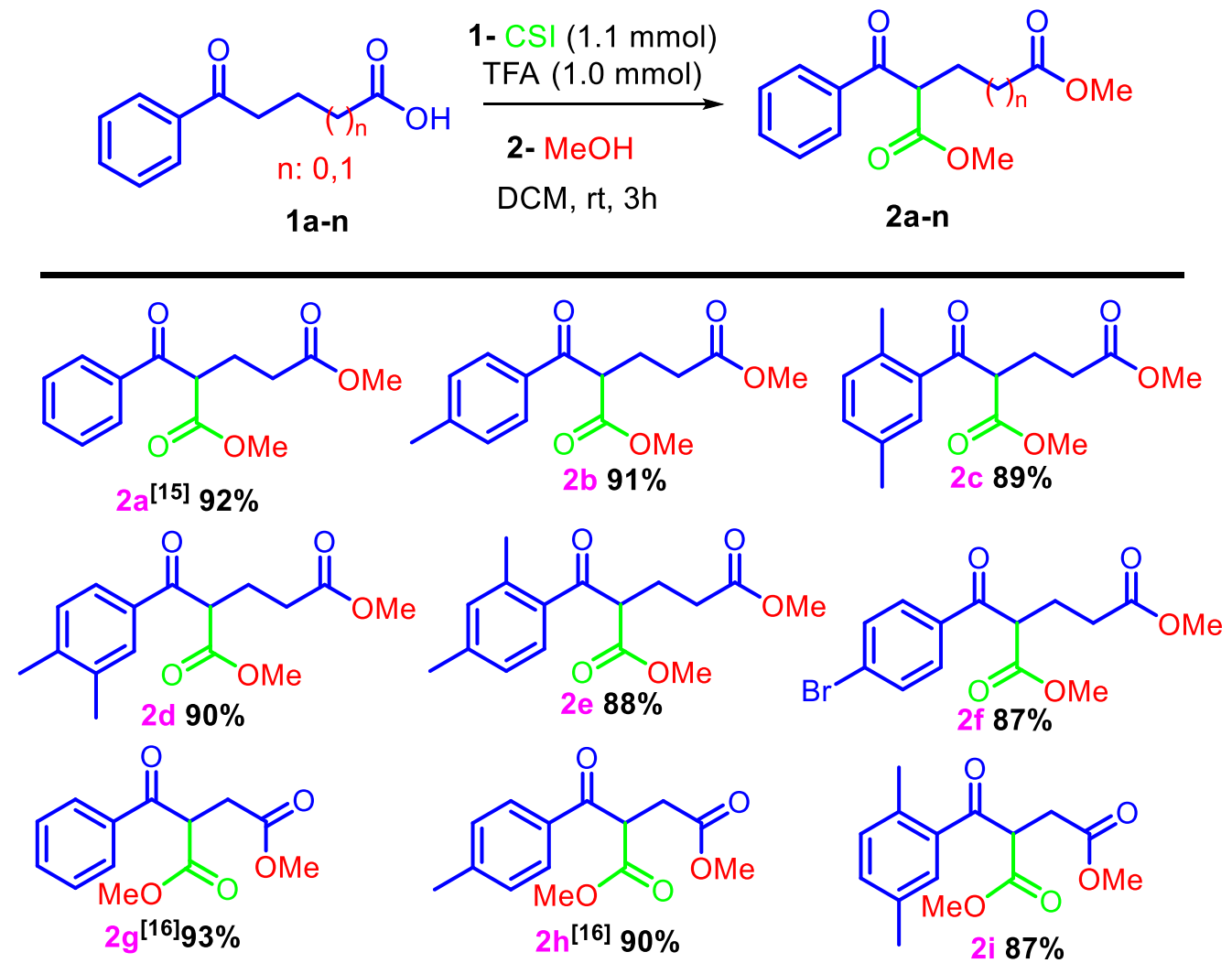<smiles>COC(=O)CC(C(=O)OC)C(=O)c1ccc(C)c(C)c1</smiles>

2j $86 \%$<smiles>COC(=O)CC(C(=O)OC)C(=O)c1ccc(C)cc1C</smiles><smiles>COC(=O)CC(C(=O)OC)C(=O)c1ccc(Br)cc1</smiles>
$\left.2\right|^{[17]} 87 \%$<smiles>COC(=O)CC(C(=O)OC)C(=O)c1ccc(I)cc1</smiles>

$2 \mathrm{~m} 84 \%$<smiles>COC(=O)CC(C(=O)OC)C(=O)c1ccc(-c2ccccc2)cc1</smiles>

2n $90 \%$

${ }^{\mathrm{a}}$ Reaction conditions: $1.0 \mathrm{mmol}$ of Carboxylic acid (1a-n), $1.0 \mathrm{mmol}$ TFA, $1.1 \mathrm{mmol}$ of CSI, $10 \mathrm{~mL} \mathrm{DCM}$, reaction was stirred for $2 \mathrm{~h}$ and added $2 \mathrm{~mL}$ $\mathrm{MeOH}$ for $1 \mathrm{~h}$. ${ }^{\mathrm{b}}$ Isolated yield. ' $\mathrm{cit}$.

With the above optimal reaction conditions in hand, we examined the scope of 4- and 5-oxo carboxylic acid derivatives (1a-n) as the reaction partners. As shown in Table 2, the reactions of 4- and 5-oxo carboxylic acid derivatives bearing electron donating groups (4-methyl, 2,5-dimethyl, 3,4-dimethyl, 2,4-dimethyl and, 4phenyl) with CSI proceeded smoothly to furnish $2 \mathrm{a}^{16}(92 \%), \mathbf{2 b}(91 \%), \mathbf{2 c}(89 \%), 2 \mathrm{~d}(90 \%), 2 \mathrm{e}(88 \%), 2 \mathrm{~g}[16]$ (93\%), $\mathbf{2} \mathbf{h}^{17}(90 \%), \mathbf{2} \mathbf{i}(87 \%), \mathbf{2} \mathbf{j}(86 \%), \mathbf{2} \mathbf{k}(86 \%)$, and $\mathbf{2 n}(90 \%)$, respectively. The halogen substituted $4-$ and $5-$ oxo-carboxylic acid derivatives $(4-\mathrm{Br}, 4-1)$ were observed also good reactivities, and the novel $\beta$-keto ester derivative $\mathbf{2 f}$ was obtained in $87 \%$ yield. $2 \mathrm{I}^{18}$ and $\mathbf{2 m}$ were isolated in $87 \%$ and $84 \%$ yield, respectively, when 4 (4-bromophenyl)-4-oxobutanoic acid (1I) and 4-(4-iodophenyl)-4-oxobutanoic acid (1 $\mathbf{m}$ ) were subjected to the reaction with CSI. 


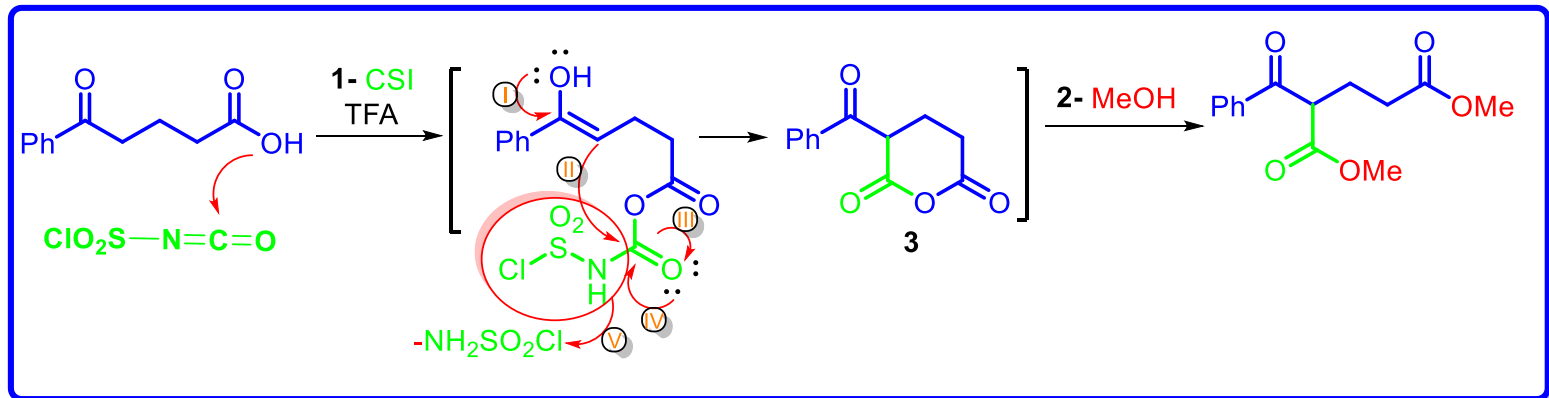

Scheme 2. Possible mechanism for synthesis of $\beta$-keto ester.

In the first step, $\mathrm{OH}$ group of carboxylic acid attacks to carbonyl group of isocyanate as nucleophilic. Intermadiate carbamate is being tautomerization (I) and following the intramolecular attack of $\alpha$-carbon to carbonyl carbamate (II, III and IV). Then, sulfamoyl chloride $\left(\mathrm{NH}_{2} \mathrm{SO}_{2} \mathrm{Cl}\right.$ ) leave from relating molecule to form cyclic anhydride (V). Finally, $\beta$-keto ester was occured by ring opening reaction of cyclic anhydride (3).

\section{Conclusions}

A pratical novel alternative method, one-pot synthesis $\beta$-keto esters with chlorosulfonyl isocyanate from 4and 5-oxocarboxylic acid derivatives, were developed in high yields under mild conditions. This novel method highly useful environmentally and benign for organic chemists and pharmaceutical industry without any metal or oxidations, and easy to handle, under mild conditions, short reaction time. Moreover, a wide variety of $\beta$ keto esters can be obtained in good yields.

\section{Experimental Section}

General. Solvents are commercially available and used without further purification. 4- and 5-oxo-carboxylic acid derivatives were synthesized as in the literature. ${ }^{19}{ }^{1} \mathrm{H}$ and ${ }^{13} \mathrm{C} N M R$ spectra were recorded a Bruker 400 $\mathrm{MHz}$ in $\mathrm{CDCl}_{3}$ with and NMR shifts are presented as $\delta$ in ppm. FTIR spectras were mesaured with a Perkin Elmer spectrophotometers in $\mathrm{CH}_{2} \mathrm{Cl}_{2}$ and by solutions in $0.1 \mathrm{~mm}$ cells. High resolution mass spectra (HRMS) were obtained with a AB-Sciex 4600 QTOF MS spectrometer.

General procedure Synthesis of $\boldsymbol{\beta}$-Keto ester. Carboxylic acid (1a-n) (1.0 eq) was dissolved in $10 \mathrm{~mL}$ DCM. The reaction mixture was added CSI (1.1 eq) and TFA (1.0 eq) and stirred for $2 \mathrm{~h}$ at room temperature. Then, it was added $2 \mathrm{~mL} \mathrm{MeOH}$ and stirred for $1 \mathrm{~h}$. The reaction mixture was extracted with dichloromethane. The organic extract was dried over sodium sulfate, filtrate and evaporation in vacuo. The resulting residue was purified by thin-layer chromatography (TLC) on silica gel.

Dimethyl 2-benzoylpentanedioate (2a). Yellowish oil (316 mg, yield 92\%), ${ }^{1} \mathrm{H}-\mathrm{NMR}\left(\mathrm{CDCl}_{3}, \mathrm{ppm}, 400 \mathrm{MHz}\right): \delta$ 2.30-2.35 (m, 2H), 2.46-2.50 (m, 2H), $3.69(\mathrm{~s}, 3 \mathrm{H}), 3.71(\mathrm{~s}, 3 \mathrm{H}), 4.55(\mathrm{t}, J 7.2 \mathrm{~Hz}, 1 \mathrm{H}), 7.50-7.53(\mathrm{~m}, 2 \mathrm{H}), 7.60-$ $7.64(\mathrm{~m}, 1 \mathrm{H}), 8.04-8.06(\mathrm{~m}, 2 \mathrm{H}) ;{ }^{13} \mathrm{C}-\mathrm{NMR}\left(\mathrm{CDCl}_{3}, \mathrm{ppm}, 100 \mathrm{MHz}\right): \delta 24.0,31.2,51.7,52.5,52.6,128.7,128.8$, 133.7, 135.9, 170.0, 173.2, 194.9; IR $\left(\mathrm{CH}_{2} \mathrm{Cl}_{2}, \mathrm{~cm}^{-1}\right)$ : 3003, 2955, 2849, 1737, 1689, 1596, 1436, 1333, 1272, 1161; HRMS (ESI) calcd. for $\mathrm{C}_{14} \mathrm{H}_{15} \mathrm{O}_{5}[\mathrm{M}-\mathrm{H}]^{-}$263.0925; found: 263.0916. 
Dimethyl 2-(4-methylbenzoyl)pentanedioate (2b). Yellowish oil (305 mg, yield 91\%), ${ }^{1} \mathrm{H}-\mathrm{NMR}\left(\mathrm{CDCl}_{3}, \mathrm{ppm}\right.$, $400 \mathrm{MHz}): \delta$ 2.28-2.32 (m, 2H), 2.42-2.47 (m, 5H), $3.67(\mathrm{~s}, 3 \mathrm{H}), 3.68(\mathrm{~s}, 3 \mathrm{H}), 4.50(\mathrm{t}, J 7.2 \mathrm{~Hz}, 1 \mathrm{H}), 7.28(\mathrm{~d}, J 8.0$, 2H), $7.92(\mathrm{~d}, J 8.0 \mathrm{~Hz}, 2 \mathrm{H}) ;{ }^{13} \mathrm{C}-\mathrm{NMR}\left(\mathrm{CDCl}_{3}, \mathrm{ppm}, 100 \mathrm{MHz}\right): \delta 21.7,24.0,51.7,52.4,52.5,128.9,129.5,133.4$, 144.7, 170.1, 173.2, 194.4; IR $\left(\mathrm{CH}_{2} \mathrm{Cl}_{2}, \mathrm{~cm}^{-1}\right):$ 3010, 2942, 2855, 1748, 1690, 1592, 1440, 1328, 128, 1153; HRMS (ESI-) calcd. for $\mathrm{C}_{14} \mathrm{H}_{15} \mathrm{O}_{5}[\mathrm{M}-\mathrm{H}]^{-}$263,0925; found: 263,0916; HRMS (ESI) calcd. for $\mathrm{C}_{15} \mathrm{H}_{17} \mathrm{O}_{5}\left[\mathrm{M}-\mathrm{H}^{-}\right.$ 277.1081; found: 277.1088.

Dimethyl 2-(2,5-dimethylbenzoyl)pentanedioate (2c). Yellowish oil (294 mg, yield 89\%), ${ }^{1} \mathrm{H}-\mathrm{NMR}\left(\mathrm{CDCl}_{3}, \mathrm{ppm}\right.$, $400 \mathrm{MHz}): \delta$ 2.25-2.28 (m, 2H), 2.35-2.44 (m, 8H), $3.67(\mathrm{~s}, 6 \mathrm{H}), 4.40(\mathrm{t}, J 7.1 \mathrm{~Hz}, 1 \mathrm{H}), 7.12-7.15(\mathrm{~m}, 1 \mathrm{H}), 7.19-$ $7.21(\mathrm{~m}, 1 \mathrm{H}), 7.49-7.51(\mathrm{~m}, 1 \mathrm{H}) ;{ }^{13} \mathrm{C}-\mathrm{NMR}\left(\mathrm{CDCl}_{3}, \mathrm{ppm}, 100 \mathrm{MHz}\right): \delta 20.6,23.9,31.3,33.1,51.7,52.4,54.9$, 129.2, 132.0, 132.6, 135.3, 135.9, 136.7, 170.1, 173.1, 198.4; IR $\left(\mathrm{CH}_{2} \mathrm{Cl}_{2}, \mathrm{~cm}^{-1}\right): 2953,1739,1652,1437,1302$, 1203, 1118; HRMS (ESI) calcd. for $\mathrm{C}_{16} \mathrm{H}_{19} \mathrm{O}_{5}[\mathrm{M}-\mathrm{H}]^{-}$291.1238; found: 291.1240.

Dimethyl 2-(3,4-dimethylbenzoyl)pentanedioate (2d). Yellowish oil (297 mg, yield 90\%), ${ }^{1} \mathrm{H}-\mathrm{NMR}\left(\mathrm{CDCl}_{3}, \mathrm{ppm}\right.$, $400 \mathrm{MHz}): \delta 2.26-2.34(\mathrm{~m}, 8 \mathrm{H}), 2.42-2.46(\mathrm{~m}, 2 \mathrm{H}), 3.67(\mathrm{~s}, 3 \mathrm{H}), 3.68(\mathrm{~s}, 3 \mathrm{H}), 4.50(\mathrm{t}, J 7.2 \mathrm{~Hz}, 1 \mathrm{H}), 7.23(\mathrm{~d}, J 7.8$ $\mathrm{Hz}, 1 \mathrm{H}), 7.75(\mathrm{~d}, J 7.8 \mathrm{~Hz}, 1 \mathrm{H}), 7.79(\mathrm{~s}, 1 \mathrm{H}) ;{ }^{13} \mathrm{C}-\mathrm{NMR}\left(\mathrm{CDCl}_{3}, \mathrm{ppm}, 100 \mathrm{MHz}\right): \delta 19.8,20.1,24.1,31.3,51.7,52.3$, $52.5,126.5,129.8,130.0,133.8,137.3,143.5,170.2,173.2,194.7 ; \mathrm{IR}\left(\mathrm{CH}_{2} \mathrm{Cl}_{2}, \mathrm{~cm}^{-1}\right): 2956,1740,1686,1611$, 1439, 1286, 1169; HRMS (ESI) calcd. for $\mathrm{C}_{16} \mathrm{H}_{19} \mathrm{O}_{5}[\mathrm{M}-\mathrm{H}]^{-} 291.1238$; found: 291.1224.

Dimethyl 2-(2,4-dimethylbenzoyl)pentanedioate (2e). Yellowish oil (292 mg, yield 88\%), ${ }^{1} \mathrm{H}-\mathrm{NMR}\left(\mathrm{CDCl}_{3}, \mathrm{ppm}\right.$, $400 \mathrm{MHz}): \delta 2.25-2.29(\mathrm{~m}, 2 \mathrm{H}), 2.33-2.48(\mathrm{~m}, 8 \mathrm{H}), 3.66(\mathrm{~s}, 3 \mathrm{H}), 3.68(\mathrm{~s}, 3 \mathrm{H}), 4.41(\mathrm{t}, J 7.2 \mathrm{~Hz}, 1 \mathrm{H}), 7.08(\mathrm{~s}, 1 \mathrm{H})$, $7.10(\mathrm{~s}, 1 \mathrm{H}), 7.66(\mathrm{~d}, \mathrm{~J} 7.8 \mathrm{~Hz}, 1 \mathrm{H}) ;{ }^{13} \mathrm{C}-\mathrm{NMR}\left(\mathrm{CDCl}_{3}, \mathrm{ppm}, 100 \mathrm{MHz}\right): \delta 21.4,24.0,51.7,52.4,54.6,126.5,129.3$, 133.1, 133.7, 139.7, 142.7, 170.2, 142.7, 170.3, 173.2, 197.5; IR $\left(\mathrm{CH}_{2} \mathrm{Cl}_{2}, \mathrm{~cm}^{-1}\right): 2953,1742,1663,1442,1328$, 1243, 1136; HRMS (ESI) calcd. for $\mathrm{C}_{16} \mathrm{H}_{19} \mathrm{O}_{5}[\mathrm{M}-\mathrm{H}]^{-}$291.1238; found: 291.1215.

Dimethyl 2-(4-bromobenzoyl)pentanedioate (2f). Yellowish oil (275 mg, yield 87\%), ${ }^{1} \mathrm{H}-\mathrm{NMR}\left(\mathrm{CDCl}_{3}, \mathrm{ppm}, 400\right.$ $\mathrm{MHz}): \delta 2.26-2.30(\mathrm{~m}, 2 \mathrm{H}), 2.43-2.47(\mathrm{~m}, 2 \mathrm{H}), 3.67(\mathrm{~s}, 3 \mathrm{H}), 3.69(\mathrm{~s}, 3 \mathrm{H}), 4.49(\mathrm{t}, J 7.2 \mathrm{~Hz}, 1 \mathrm{H}), 7.63(\mathrm{~d}, J 8.5 \mathrm{~Hz}$, 2H), 7.90 (d, J $8.5 \mathrm{~Hz}, 2 \mathrm{H}) ;{ }^{13} \mathrm{C}-\mathrm{NMR}\left(\mathrm{CDCl}_{3}, \mathrm{ppm}, 100 \mathrm{MHz}\right): \delta 23.4,31.1,33.0,51.7,52.4,129.1,130.2,132.2$, 134.6, 169.8, 173.2, 193.9; IR $\left(\mathrm{CH}_{2} \mathrm{Cl}_{2}, \mathrm{~cm}^{-1}\right): 2952,1735,1686,1583,1435,1329,1272,1171$; HRMS (ESI) calcd. for $\mathrm{C}_{14} \mathrm{H}_{14} \mathrm{BrO}_{5}[\mathrm{M}-\mathrm{H}]^{-}$341.0030; found: 341.0035 .

Dimethyl 2-benzoylsuccinate (2g). Yellowish oil (327 mg, yield 93\%), ${ }^{1} \mathrm{H}-\mathrm{NMR}\left(\mathrm{CDCl}_{3}, \mathrm{ppm}, 400 \mathrm{MHz}\right): \delta 3.03-$ $3.14(\mathrm{~m}, 2 \mathrm{H}), 3.67(\mathrm{~s}, 3 \mathrm{H}), 3.68(\mathrm{~s}, 3 \mathrm{H}), 4.89(\mathrm{t}, J 7.2 \mathrm{~Hz}, 1 \mathrm{H}), 7.48(\mathrm{~d}, J 7.8 \mathrm{~Hz}, 2 \mathrm{H}), 7.58-7.61(\mathrm{~m}, 1 \mathrm{H}), 8.04(\mathrm{~d}, J$ $7.3 \mathrm{~Hz}, 2 \mathrm{H}) ;{ }^{13} \mathrm{C}-\mathrm{NMR}\left(\mathrm{CDCl}_{3}, \mathrm{ppm}, 100 \mathrm{MHz}\right): \delta 33.1,49.3,52.1,52.9,128.8,128.9,133.8,135.8,169.2,171.7$, 194.0; IR $\left(\mathrm{CH}_{2} \mathrm{Cl}_{2}, \mathrm{~cm}^{-1}\right)$ : 3003, 2955, 2849, 1737, 1689, 1596, 1436, 1333, 1272, 1161; HRMS (ESI) calcd. for $\mathrm{C}_{13} \mathrm{H}_{14} \mathrm{O}_{5}[\mathrm{M}-\mathrm{H}]^{-} 250.0847$; found: 250.0832.

Dimethyl 2-(4-methylbenzoyl)succinate (2h). Yellowish oil (309 mg, yield 90\%), ${ }^{1} \mathrm{H}-\mathrm{NMR}\left(\mathrm{CDCl}_{3}, \mathrm{ppm}, 400\right.$ $\mathrm{MHz}): \delta 2.42(\mathrm{~s}, 3 \mathrm{H}), 3.05-3.08(\mathrm{~m}, 2 \mathrm{H}), 3.67(\mathrm{~s}, 3 \mathrm{H}), 3.69(\mathrm{~s}, 3 \mathrm{H}), 4.87(\mathrm{t}, J 7.2 \mathrm{~Hz}, 1 \mathrm{H}), 7.29(\mathrm{~d}, J 8.2 \mathrm{~Hz}, 2 \mathrm{H})$, $7.93(\mathrm{~d}, J 8.2 \mathrm{~Hz}, 2 \mathrm{H}) ;{ }^{13} \mathrm{C}-\mathrm{NMR}\left(\mathrm{CDCl}_{3}, \mathrm{ppm}, 100 \mathrm{MHz}\right): \delta 28.9,33.1,49.2,52.1,52.8,129.1,129.5,133.3$, 144.8, 169.3, 171.8, 193.5; IR $\left(\mathrm{CH}_{2} \mathrm{Cl}_{2}, \mathrm{~cm}^{-1}\right)$ : 2953, 2826, 1737, 682, 1607, 1436, 1255, 1165; HRMS (ESI) calcd. for $\mathrm{C}_{14} \mathrm{H}_{15} \mathrm{O}_{5}[\mathrm{M}-\mathrm{H}]^{-}$263.0925; found: 263.0904 .

Dimethyl 2-(2,5-dimethylbenzoyl)succinate (2i). Yellowish oil (293 mg, yield 87\%), ${ }^{1} \mathrm{H}-\mathrm{NMR}\left(\mathrm{CDCl}_{3}, \mathrm{ppm}, 400\right.$ $\mathrm{MHz}): \delta 2.37(\mathrm{~s}, 3 \mathrm{H}), 2.41(\mathrm{~s}, 3 \mathrm{H}), 3.01-3.11(\mathrm{~m}, 2 \mathrm{H}), 3.67(\mathrm{~s}, 3 \mathrm{H}), 3.69(\mathrm{~s}, 3 \mathrm{H}), 4.76(\mathrm{t}, J \mathrm{~J} .2 \mathrm{~Hz}, 1 \mathrm{H}), 7.23-7.25$ $(\mathrm{m}, 1 \mathrm{H}), 7.76-7.80(\mathrm{~m}, 2 \mathrm{H}) ;{ }^{13} \mathrm{C}-\mathrm{NMR}\left(\mathrm{CDCl}_{3}, \mathrm{ppm}, 100 \mathrm{MHz}\right): \delta 20.4,21.0,32.9,51.9,52.1,52.7,129.3,131.9$, 132.6, 135.3, 135.9, 136.7, 169.3, 171.8, 197.5; IR $\left(\mathrm{CH}_{2} \mathrm{Cl}_{2}, \mathrm{~cm}^{-1}\right): 2951,1734,1680,1432,1272,1170 ; \mathrm{HRMS}$ (ESI) calcd. for $\mathrm{C}_{15} \mathrm{H}_{17} \mathrm{O}_{5}[\mathrm{M}-\mathrm{H}]^{-}$277.1081; found: 277.1090.

Dimethyl 2-(3,4-dimethylbenzoyl)succinate (2j). Yellowish oil (290 mg, yield 86\%), ${ }^{1} \mathrm{H}-\mathrm{NMR}\left(\mathrm{CDCl}_{3}, \mathrm{ppm}, 400\right.$ $\mathrm{MHz}): \delta 2.32(\mathrm{~s}, 6 \mathrm{H}), 2.98-3.11(\mathrm{~m}, 2 \mathrm{H}), 3.67(\mathrm{~s}, 3 \mathrm{H}), 3.69(\mathrm{~s}, 3 \mathrm{H}), 4.87(\mathrm{t}, J 7.2 \mathrm{~Hz}, 1 \mathrm{H}), 7.11-7.22(\mathrm{~m}, 2 \mathrm{H}), 7.58$ $(\mathrm{s}, 1 \mathrm{H}) ;{ }^{13} \mathrm{C}-\mathrm{NMR}\left(\mathrm{CDCl}_{3}, \mathrm{ppm}, 100 \mathrm{MHz}\right): \delta 19.8,20.1,33.1,49.1,52.1,52.8,126.7,129.9,130.0,133.6,137.2$, 
143.6, 169.4, 171.8, 193.8; IR $\left(\mathrm{CH}_{2} \mathrm{Cl}_{2}, \mathrm{~cm}^{-1}\right)$ : 2954, 1738, 1684, 1605, 1438, 1276, 1174; HRMS (ESI) calcd. for $\mathrm{C}_{15} \mathrm{H}_{17} \mathrm{O}_{5}[\mathrm{M}-\mathrm{H}]^{-}$277.1081; found: 277.1054 .

Dimethyl 2-(2,4-dimethylbenzoyl)succinate (2k). Yellowish oil (291 mg, yield 86\%), ${ }^{1} \mathrm{H}-\mathrm{NMR}\left(\mathrm{CDCl} \mathrm{l}_{3} \mathrm{ppm}, 400\right.$ $\mathrm{MHz}): \delta 2.35(\mathrm{~s}, 3 \mathrm{H}), 2.46(\mathrm{~s}, 3 \mathrm{H}), 2.94-3.11(\mathrm{~m}, 2 \mathrm{H}), 3.67(\mathrm{~s}, 3 \mathrm{H}), 3.68(\mathrm{~s}, 3 \mathrm{H}), 4.77(\mathrm{t}, J 6.6 \mathrm{~Hz}, 1 \mathrm{H}), 7.08-7.11$ $(\mathrm{m}, 2 \mathrm{H}), 7.74(\mathrm{~d}, J \mathrm{7.8}, \mathrm{Hz}, 1 \mathrm{H}) ;{ }^{13} \mathrm{C}-\mathrm{NMR}\left(\mathrm{CDCl}_{3}, \mathrm{ppm}, 100 \mathrm{MHz}\right): \delta 21.3,21.4,33.1,51.6,52.1,52.7,126.4$, 129.4, 133.0, 133.7, 139.6, 142.7, 169.5, 171.8, 196.6; IR $\left(\mathrm{CH}_{2} \mathrm{Cl}_{2}, \mathrm{~cm}^{-1}\right): 2955,2893,1741,1683,1439,1203$, 1173; HRMS (ESI) calcd. for $\mathrm{C}_{15} \mathrm{H}_{17} \mathrm{O}_{5}[\mathrm{M}-\mathrm{H}]^{-}$277.1081; found: 277.1074 .

Dimethyl 2-(4-bromobenzoyl)succinate (2l). Yellowish oil $(279 \mathrm{mg}$, yield $87 \%),{ }^{1} \mathrm{H}-\mathrm{NMR}\left(\mathrm{CDCl}_{3}, \mathrm{ppm}, 400\right.$ $\mathrm{MHz}): \delta 3.01-3.17(\mathrm{~m}, 2 \mathrm{H}), 3.67(\mathrm{~s}, 3 \mathrm{H}), 3.68(\mathrm{~s}, 3 \mathrm{H}), 4.82(\mathrm{dd}, J 6.2,8.1 \mathrm{~Hz}, 1 \mathrm{H}), 7.63-7.65(\mathrm{~m}, 2 \mathrm{H}), 7.89-7.92$ $(\mathrm{m}, 2 \mathrm{H}) ;{ }^{13} \mathrm{C}-\mathrm{NMR}\left(\mathrm{CDCl}_{3}, \mathrm{ppm}, 100 \mathrm{MHz}\right): \delta 33.0,49.1,52.2,53.0,129.1,130.4,132.1,134.6,168.8,171.7$, 193.1; IR $\left(\mathrm{CH}_{2} \mathrm{Cl}_{2}, \mathrm{~cm}^{-1}\right)$ : 2954, 1737, 1688, 1585, 1437, 1331, 1274, 1173; HRMS (ESI) calcd. for $\mathrm{C}_{13} \mathrm{H}_{12} \mathrm{BrO}_{5}[\mathrm{M}-$ H] 326.9874 ; found: 326.9863.

Dimethyl 2-(4-iodobenzoyl)succinate (2m). Yellowish oil (260 mg, yield 84\%), ${ }^{1} \mathrm{H}-\mathrm{NMR}\left(\mathrm{CDCl}_{3}, \mathrm{ppm}, 400 \mathrm{MHz}\right)$ :

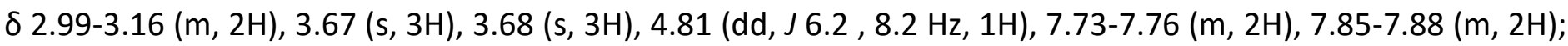
${ }^{13} \mathrm{C}-\mathrm{NMR}\left(\mathrm{CDCl}_{3}\right.$, ppm, $\left.100 \mathrm{MHz}\right): \delta 33.0,49.1,52.2,53.0,102.1,130.2,135.2,138.1,168.8,171.7,193.5 ; \mathrm{IR}$ $\left(\mathrm{CH}_{2} \mathrm{Cl}_{2}, \mathrm{~cm}^{-1}\right): 2952,2833,1737,1687,1581,1437,1272,1071$; HRMS (ESI) calcd. for $\mathrm{C}_{13} \mathrm{H}_{12} \mathrm{IO}_{5}\left[\mathrm{M}-\mathrm{H}^{-}\right.$ 374.9735; found: 374.9732 .

Dimethyl 2-([1,1'-biphenyl]-4-carbonyl)succinate (2n). Yellow solid (289 mg, yield 90\%), ${ }^{1} \mathrm{H}-\mathrm{NMR}\left(\mathrm{CDCl}_{3}, \mathrm{ppm}\right.$, $400 \mathrm{MHz}): \delta 3.03-3.18(\mathrm{~m}, 2 \mathrm{H}), 3.68(\mathrm{~s}, 3 \mathrm{H}), 3.70(\mathrm{~s}, 3 \mathrm{H}), 4.93(\mathrm{t}, J \mathrm{~J} .2 \mathrm{~Hz}, 1 \mathrm{H}), 7.41-7.50(\mathrm{~m}, 3 \mathrm{H}), 7.62-7.73(\mathrm{~m}$, $4 \mathrm{H}), 8.11-8.14(\mathrm{~m}, 2 \mathrm{H}) ;{ }^{13} \mathrm{C}-\mathrm{NMR}\left(\mathrm{CDCl}_{3}, \mathrm{ppm}, 100 \mathrm{MHz}\right): \delta 33.1,49.2,52.2,52.9,127.3,127.4,128.4,129.0$, 129.6, 134.5, 139.7, 146.5, 169.2, 171.8, 193.6; IR $\left(\mathrm{CH}_{2} \mathrm{Cl}_{2}, \mathrm{~cm}^{-1}\right): 2953,1736,682,603,1436,1274,1172$; HRMS (ESI) calcd. for $\mathrm{C}_{19} \mathrm{H}_{17} \mathrm{O}_{5}[\mathrm{M}-\mathrm{H}]^{-} 325.1081$; found: 325.1071 .

3-Benzoyldihydro-2H-pyran-2,6(3H)-dione (3). Reaction mixture ${ }^{1} \mathrm{H}-\mathrm{NMR}\left(\mathrm{CDCl}_{3}, \mathrm{ppm}, 400 \mathrm{MHz}\right): \delta 2.31-2.47$ $(\mathrm{m}, 2 \mathrm{H}), 2.93-2.96(\mathrm{~m}, 2 \mathrm{H}), 4.92(\mathrm{t}, J 5.3 \mathrm{~Hz}, 1 \mathrm{H}), 7.53-7.59(\mathrm{~m}, 2 \mathrm{H}), 7.67-7.73(\mathrm{~m}, 1 \mathrm{H}), 7.96-7.98(\mathrm{~m}, 2 \mathrm{H})$.

\section{Acknowledgements}

We gratefully acknowledge Atatürk University, Faculty of Sciences, Department of Chemistry, for support of this work.

\section{Supplementary Material}

Experimental details, ${ }^{1} \mathrm{H}$ - and ${ }^{13} \mathrm{C}-\mathrm{NMR}$ spectra for the product compounds $\mathbf{2 a}-\mathbf{2} \mathbf{n}$ and $\mathbf{3}$ are provided in Supplementary Material file on the online version of the text.

\section{References}

1. Benetti, S.; Romagnoli, R.; De Risi, C.; Spalluto, G.; Zanirato, V. Chem. Rev. 1995, 95, 1065-1114. https://doi.org/10.1021/cr00036a007

2. Urban, E.; Knühl, G.; Helmchenb, G. Tetrahedron 1995, 51(47), 13031-13038. 
3. Benbakkar, M.; Baltas, M.; Gorrichon, J. P. Synth. Commun. 1989, 19(18), 3241-3247. https://doi.org/10.1080/00397918908052724

4. Matsuura, F.; Hamada, Y.; Shioiri, T. Tetrahedron Lett. 1994, 35(5), 733. https://doi.org/10.1016/S0040-4039(00)75803-5

5. Bestmann, H. J.; Kolm, H. Chem. Ber. 1963, 96(7), 1948-1958. https://doi.org/10.1002/cber.19630960730

6. Stork, G.; Brizzolara, A.; Landesman, H.; Szmuszkovich, J.; Terrell, R. J. Am. Chem. Soc. 1963, 85(2), 207222.

https://doi.org/10.1021/ja00885a021

7. Lawesson, S. O.; Gonwall, S.; Sandberg, R. Org. Synth. 1962, 42, 28.

https://doi.org/10.15227/orgsyn.042.0028

8. Hauser, C. R.; Hudson, B. E. Jr. Org. React. 1942, 1, 266.

9. Snyder, H. R.; Brooks, L. A.; Shapiro, S. H. Org. Synth. Coll. 1943, 2; Wiley: New York, 531.

10. Krapcho, A. P.; Diamanti, J.; Cayen, C.; Bingham, R. Org. Synth. 1967, 47, 20. https://doi.org/10.15227/orgsyn.047.0020

11. Lapidus, A. L.; Eliseev, O. L.; Bondarenko, T. N.; Sizan, O. E.; Ostapenko, A. G.; Beletskaya, I. P. Synthesis 2002, 3(18), 317-319.

https://doi.org/10.1055/s-2002-20027

12. Yadav, J. S.; Subba Reddy, B. V.; Eeshwaraiah, B.; Reddy, P. N. Tetrahedron 2005, 61, 875-878. https://doi.org/10.1016/j.tet.2004.11.027

13. Huang, P. Q.; Ou, W. Eur. J. Org. Chem. 2017, 582-592. https://doi.org/10.1002/ejoc.201601326

14. Rasmussen, J. K.; Hassner, A. Chem. Rev. 1976, 76(3) 389-408. https://doi.org/10.1021/cr60301a004

15. Atmaca, U. Tetrahedron 2019, 75(34), 130467. https://doi.org/10.1016/j.tet.2019.130467

16. Wahl, B.; Philipson, Y.; Bonin, H.; Mortreux, A.; Sauthier, M. J. Org. Chem. 2013, 78, 1547-1552. https://doi.org/10.1021/j03026143

17. Rohe, S.; Morris, A. O.; McCallum, T.; Barriault, L. Angew. Chem. Int. Ed. 2018, 130(48), 15890-15895. https://doi.org/10.1002/ange.201810187

18. Vu, M. D.; Das, M.; Liu X. W. Chem. Eur. J. 2017, 23, 15899 - 15902. https://doi.org/10.1002/chem.201704224

19. Cai, J.; Liu, L.; Hong, K. H.; Wang, P.; Li, L.; Cao, M.; Sun, C.; Wu, X.; Zong, X.; Chen, J.; Ji, M. Bioorg. Med. Chem. 2015, 23, 65767. https://doi.org/10.1016/j.bmc.2015.01.003 